\title{
A Graphite-Polysulfide Full Cell with DME-Based Electrolyte
}

\author{
Amruth Bhargav, ${ }^{\mathrm{a}, *}$ Min $\mathrm{Wu}^{\text {, }}$ and Yongzhu $\mathrm{Fu}^{\mathrm{a}, \mathrm{b}, * *, \mathrm{z}}$ \\ ${ }^{a}$ Department of Mechanical Engineering, Indiana University-Purdue University Indianapolis, Indianapolis, \\ Indiana 46202, USA \\ ${ }^{b}$ Richard G. Lugar Center for Renewable Energy, Indiana University-Purdue University Indianapolis, Indianapolis, \\ Indiana 46202, USA
}

\begin{abstract}
Over the last decade, vast improvements have been made in the field of lithium-sulfur batteries bringing it a step closer to reality. In this field of research, deep understanding of the polysulfide shuttle phenomenon and their affinity with carbons, polymers and other hosts have enabled the design of superior cathodes with prolonged life. However, the anode side has undergone comparatively less transformation. In this work, we have developed a new electrolyte based on 1,2-dimethoxyethane (DME) solvent that enables reversible intercalation of lithium ions in graphite. A novel method to introduce solid lithium polysulfide into a carbon current collector as the cathode has been demonstrated and the electrode shows stable cycling with the new electrolyte. A full cell consisting of a lithiated graphitic anode and lithium polysulfide cathode is constructed, which exhibits an initial capacity as high as 1,500 $\mathrm{mAh} \mathrm{g}^{-1}$ (based on the sulfur in the cathode) and a reversible capacity of $700 \mathrm{mAh} \mathrm{g}^{-1}$ for 100 cycles. This full cell is capable of delivering over $460 \mathrm{mAh} \mathrm{g}^{-1}$ at rates as high as $2 C$. The cell degradation over prolonged cycles could be due to the polysulfide shuttle which results in instability of the SEI layer on the graphitic anode.

(C) The Author(s) 2016. Published by ECS. This is an open access article distributed under the terms of the Creative Commons Attribution 4.0 License (CC BY, http://creativecommons.org/licenses/by/4.0/), which permits unrestricted reuse of the work in any medium, provided the original work is properly cited. [DOI: 10.1149/2.0151608jes] All rights reserved.
\end{abstract}

Manuscript submitted April 11, 2016; revised manuscript received May 3, 2016. Published May 19, 2016. This article is a version of Paper 333 from the San Diego, California, Meeting of the Society, May 29-June 2, 2016.

The demand for energy consumption by mankind is ever increasing due to rapid growth and accessibility of technology by the masses. This has led to our dependence on fossil fuels like coal and petroleum. Fortunately, sulfur, one of the promising cathode materials for inexpensive high energy density lithium batteries arises as a by-product of petroleum refining. ${ }^{1}$ Its abundant, benign nature combined with the ability of lithium-sulfur (Li-S) cells to provide a theoretical specific capacity of 1,672 $\mathrm{mAh} \mathrm{g}^{-1}$ and specific energy of $\sim 2,600 \mathrm{Wh} \mathrm{kg}^{-1}$ makes it an attractive cathode material. ${ }^{2}$ With high promises come significant challenges in utilizing this material effectively toward commercialization. The significant ones being the low conductivity of sulfur and lithium sulfide, the shuttle effect caused by the mobile intermediate polysulfides, and the volume changes upon cycling in the cathode. $^{2-4}$ In recent years, most of the research efforts have been focused to tackle issues at the cathode side. The pure lithium metal used in the cell also poses crucial challenges in the development of Li-S systems. Chief among them being the formation of $\mathrm{Li}$ dendrites and mossy deposits on the $\mathrm{Li}$ anode, ${ }^{5,6}$ presence of excess lithium which assists the shuttle effect, ${ }^{7}$ and low Coulombic efficiency associated with Li metal deposition and stripping which leads to short cycle life. ${ }^{5}$ To overcome the shortcomings on the anode side different nonLi metal anodes have been tested such as graphite, ${ }^{8-11}$ hard carbon, ${ }^{12}$ silicon, ${ }^{13,14}$ tin, ${ }^{15}$ and other alloys. ${ }^{8}$ Although non-lithium anodes prevent Li-dendrite formation and increase Coulombic efficiency at the anode side, it is imperative that a compatible electrolyte that can form a stable solid electrolyte interphase (SEI) and promote high anode capacity is used. The specific capacity provided by the graphite anode while using the common electrolyte of $1 \mathrm{M}$ LiTFSI in DME/DOL is much lower than the carbonate electrolyte for Li-ion batteries, i.e., $1 \mathrm{M} \mathrm{LiPF}_{6}$ in EC/DEC. ${ }^{11}$ This provides the motivation to develop alternative electrolytes that work with graphite while utilizing higher anode capacity that facilitates higher energy density.

In this regard, recent work on high concentration electrolytes have shown to offer stable Li intercalation into graphite. ${ }^{16}$ Not only this, high electrolyte concentration effectively suppresses the lithium polysulfide shuttle effect when coupled with Li-S batteries. ${ }^{17,18}$ In this work, we have incorporated a new high concentration electrolyte based on a combination of $3 \mathrm{M}$ lithium bis(fluorosulfonyl)imide (LiFSI, $\left.\mathrm{LiN}\left(\mathrm{SO}_{2} \mathrm{~F}\right)_{2}\right)$ and $1 \mathrm{M}$ lithium bis(trifluoromethane sulfonyl) imide

\footnotetext{
*Electrochemical Society Student Member

**Electrochemical Society Member.

${ }^{\mathrm{z} E}$-mail: yongfu@iupui.edu
}

(LiTFSI, $\left.\mathrm{LiN}\left(\mathrm{SO}_{2} \mathrm{CF}_{3}\right)_{2}\right)$ in 1,2-dimethoxyethane (DME) as the solvent. This electrolyte uses the beneficial effects of both the LiFSI and LiTFSI salts while supporting Li intercalation at the anode and suppressing polysulfide shuttle from the cathode. ${ }^{19-21}$ Full cells were made with a novel lithium polysulfide cathode and MCMB based anode. The cells exhibit good cycle performance and high rate capability. The morphological changes at both the cathode and anode before and after cycling were studied to better understand the lithium-metal-free Li-S full cells.

\section{Experimental}

Anode preparation.-A slurry containing $80 \mathrm{wt} \%$ MesoCarbon MicroBeads (MCMB, MTI corp.), 10 wt\% Super C65 (conductive carbon black, Timcal), and $10 \mathrm{wt} \%$ polyvinylidene fluoride (PVdF, Kureha Battery Materials Japan Co., Ltd.) with N-methyl2-pyrrolidone (NMP, Acros Organics) solvent was cast on Toray carbon paper (TGP-H-090, Fuel Cell Earth LLC) using a doctor blade. The electrode was dried overnight at $100^{\circ} \mathrm{C}$. The electrode sheet was punched into approximately $1 \mathrm{~cm}^{2}$ discs with MCMB loading of about $1.4 \mathrm{mg} \mathrm{cm}^{-2}$ and used as the anode. In this MCMB-carbon paper (MCMB-CP) hybrid anode, lithium storage capacity comes from both the MCMB and carbon paper. $50 \%$ of the weight of the carbon paper disc $(\sim 12.4 \mathrm{mg})$ consists of graphitic carbon, ${ }^{22}$ the mass of which is included in the specific capacity calculation.

Cathode preparation.-The lithium polysulfide solution was prepared by dissolving stoichiometric amounts of lithium sulfide $\left(\mathrm{Li}_{2} \mathrm{~S}\right.$, Sigma Aldrich) and sublimed sulfur (S, Fisher scientific) to form 0.25 $\mathrm{M} \mathrm{Li}_{2} \mathrm{~S}_{6}$ (corresponding to $1.5 \mathrm{M}$ sulfur) in methanol. Also, $0.75 \mathrm{M}$ $\mathrm{Li}_{2} \mathrm{~S}_{6}$ solution in ethanol was prepared to develop the higher loading cathode. The cathode consisted of $1 \mathrm{~cm}^{2}$ discs of commercial multiwall carbon nanotube (MWCNT) paper or buckypaper (NTL composites) to which $20 \mu \mathrm{L}$ of $0.25 \mathrm{M} \mathrm{Li}_{2} \mathrm{~S}_{6}$ solution was added and let to dry overnight to yield the solid lithium polysulfide cathode containing $1.0 \mathrm{mg}$ of sulfur, which was primarily used in this study. $30 \mu \mathrm{L}$ of $0.75 \mathrm{M}$ solution was used to prepare the high loading cathode containing $4.3 \mathrm{mg}$ of sulfur.

Electrochemical testing.-The primary electrolyte used in this study consists of $3 \mathrm{M}$ lithium bis(fluorosulfonyl)imide (LiFSI, Oakwood Chemical) and $1 \mathrm{M}$ lithium bis(trifluoromethanesulfonyl) imide (LiTFSI, Sigma Aldrich) in 1,2-dimethoxyethane (DME, Sigma Aldrich) as the solvent. 1 M LiTFSI in DME and 1,3-dioxolane (DOL, Sigma Aldrich) in $1: 1 \mathrm{v} / \mathrm{v}$ ratio as an electrolyte and commercial 

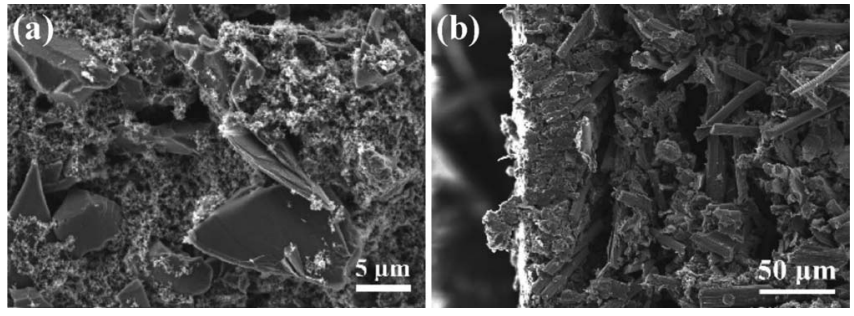

Figure 1. SEM images of the MCMB-CP anode showing (a) its face and (b) its cross-section.

Li-ion battery electrolyte, i.e. $1 \mathrm{M} \mathrm{LiPF}_{6}$ in ethylene carbonate/diethyl carbonate (EC/DEC, 1:1 v/v, Novolyte) were used in comparison studies. Half-cell tests were carried out using CR2032 type coin cells with the anode/cathode discs, $30 \mu \mathrm{L}$ of electrolyte, Celgard 2400 separator and lithium metal disc as the counter and reference electrode. The cells were cycled between $2-0.01 \mathrm{~V}$ for anode tests and between $3-1.8 \mathrm{~V}$ for cathode tests at the appropriate $C$-rate ( $C$ corresponding to $372 \mathrm{~mA} \mathrm{~g}^{-1}$ graphite for the anode and $1,672 \mathrm{~mA} \mathrm{~g}^{-1}$ sulfur for the cathode). For full cells, the MCMB-CP anode was pre-lithiated using a half-cell at $C / 15$ and the lithiated anode was extracted, washed with neat DME, and dried in the glove box atmosphere before being used as an anode. The full cell was made by coupling the polysulfide cathode with the lithiated MCMB-CP anode. Full cells were cycled at the appropriate $C$-rate ( $C$ corresponding to $1,672 \mathrm{~mA} \mathrm{~g}^{-1}$ sulfur in the cathode) between $2.8-1.85 \mathrm{~V}$. Cyclic voltammetry (CV) was performed on a Bio-Logic VSP potentiostat between $2.8-1.8 \mathrm{~V}$ at a scanning rate of $0.1 \mathrm{mV} \mathrm{s}^{-1}$.

Material characterization.-Structure and morphology change was observed using scanning electron microscopy (SEM) performed on a JEOL JSM-7800F field emission scanning electron microscope equipped with energy-dispersive X-ray spectroscopy (EDX). SEM samples were washed using DME solvent and dried before being mounted inside the glove box. The samples were transferred using an argon-filled sealed container. X-Ray Diffraction (XRD) studies used a Bruker D8 Discover diffractometer with $\mathrm{Cu} \mathrm{K} \alpha$ radiation source of wavelength $1.54184 \AA$ for $2 \theta$ between $20^{\circ}$ and $80^{\circ}$ at a scan rate of $1.25^{\circ} \mathrm{min}^{-1}$. XRD samples were prepared similar to SEM samples and covered with a Kapton film to prevent any exposure to air.

\section{Results and Discussion}

Anode and electrolyte.-The anode used in the full cell consists of MCMB graphite embedded in a matrix of carbon fibers of the carbon paper to form the MCMB-CP hybrid anode. Figure 1a shows the SEM image of the top surface of the anode. MCMB particles are deeply covered in super C65 thus ensuring good electrical contact into the graphite. The SEM image (Figure 1b) of the anode crosssection shows the MCMB-super C65 composite is present through the depth of the carbon fiber of the carbon paper. The filled pores of the crisscrossed carbon fibers ensure abundant electron transfer pathways while reducing the porosity of the anode which could reduce polysulfide retention due to shuttle effect.

XRD investigation of the pristine anode (Figure 2a) shows a distinctive sharp peak at $26.4^{\circ}$ corresponding to the (002) plane of the graphite present in both MCMB and the carbon paper. There is also a broad slope at about $26^{\circ}$ which arises due to the non-graphitic carbon fibers present in the carbon paper. On electrochemical lithiation of this anode, the peak shifts to $24.5^{\circ}$ owing to the increase in the graphite interlayer spacing due to lithium intercalation. On de-intercalation, the graphite returns to its original configuration thus showing the reversible lithium intercalation and de-intercalation supported by the 3 M LiFSI/1 M LiTFSI in DME electrolyte.

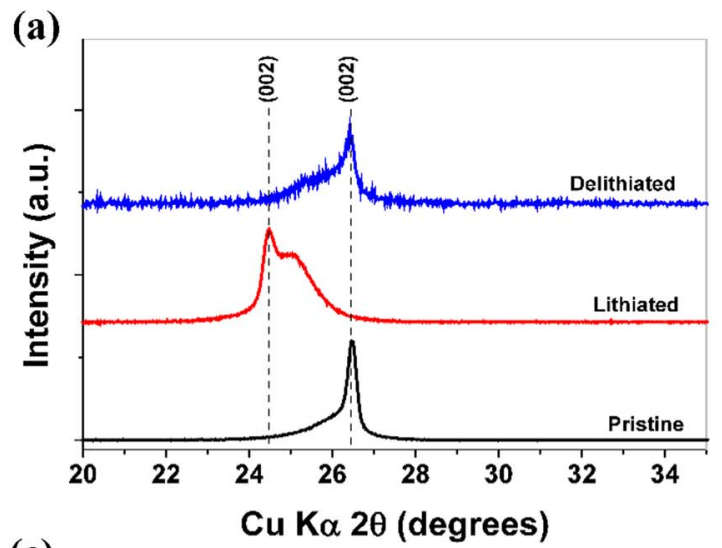

(c)

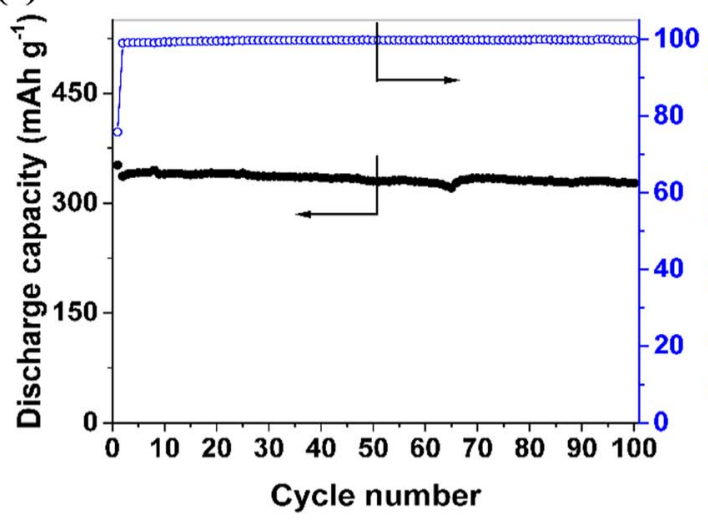

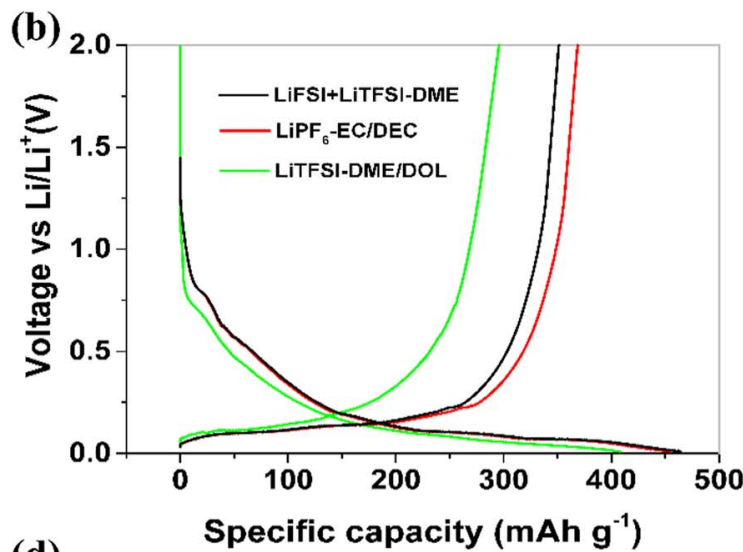

(d)

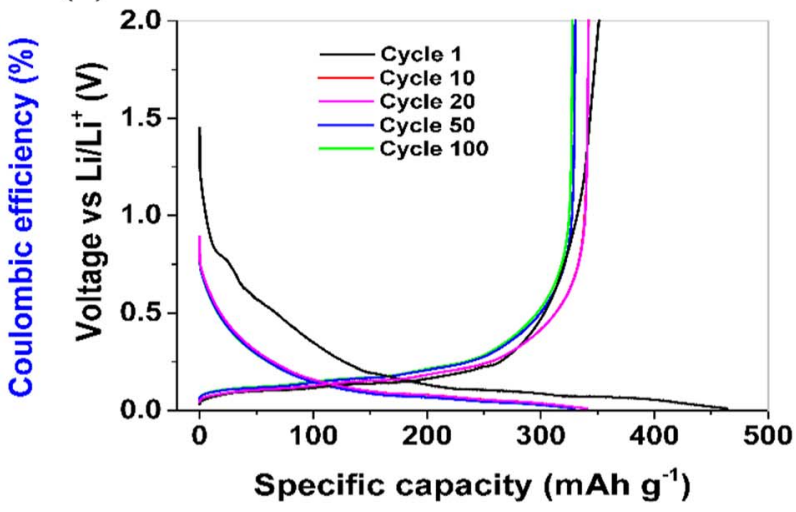

Figure 2. (a) XRD showing the intercalation behavior of MCMB-CP anode with $3 \mathrm{M} \mathrm{LiFSI}+1 \mathrm{M}$ LiTFSI in DME electrolyte, (b) first cycle performance comparing the anode performance under different electrolytes, (c) cycle life of the MCMB-CP anode with DME electrolyte along with the corresponding voltage profiles in (d). The cells were cycled at $C / 10\left(1 C=372 \mathrm{~mA} \mathrm{~g}^{-1}\right.$, based on the mass of graphite in the whole electrode). 
(a)

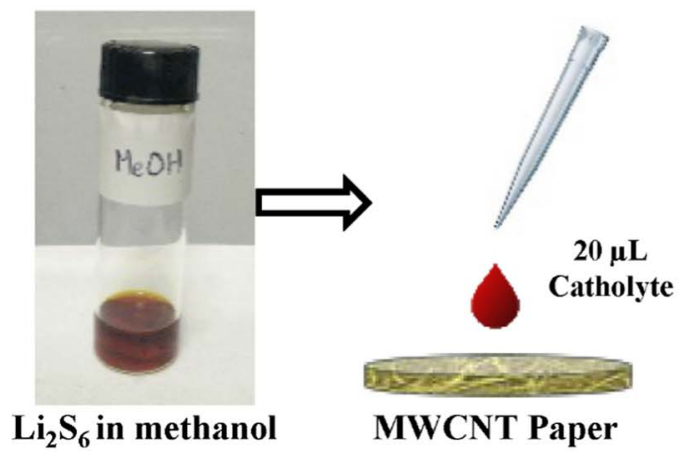

(b)

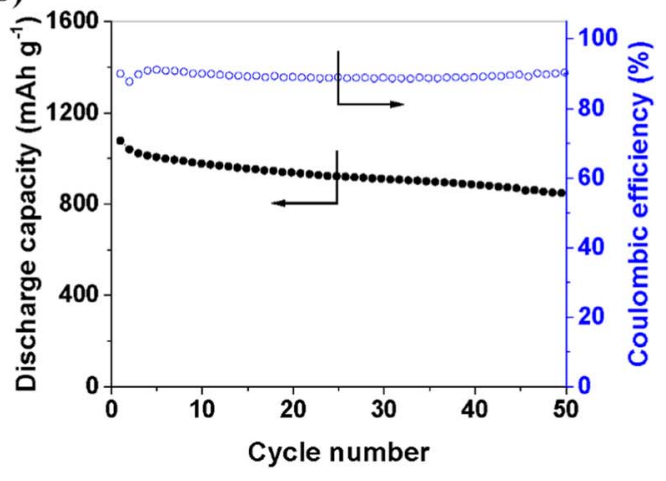

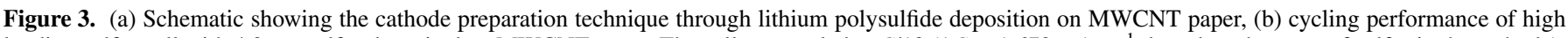

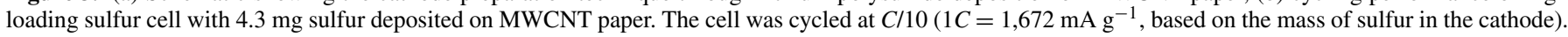

High concentration LiFSI based electrolytes have shown stable lithium intercalation. ${ }^{16}$ But, LiFSI-based electrolyte shows unfavorable performance with sulfur cathode at room temperature. ${ }^{21}$ However, the addition of a similar salt, namely, LiTFSI can stabilize the electrolyte due to common-ion effect. ${ }^{19,20}$ Thus, this unique combination of $3 \mathrm{M}$ LiFSI and $1 \mathrm{M}$ LiTFSI has been used in this study to accommodate both highly reversible lithium intercalation behavior offered by LiFSI and the stability with sulfur cathode provided by LiTFSI while utilizing DME alone as the electrolyte solvent.

To compare the performance of this newly developed electrolyte with the commercial Li-ion battery electrolyte which is known to exhibit high capacity and reversibility and the electrolyte commonly used in Li-S studies, including full cell studies, ${ }^{9,12}$ half cells with the MCMB-CP anode were tested. The first cycle performance shown in Figure $2 b$ uses reversible specific capacity delivered by the anode as a measure of the effectiveness these electrolytes. Higher specific capacity equates to lower amount of graphite required to support the capacity delivered by sulfur cathode, thus improving the specific energy of the system. It is apparent that the best performance is provided by the carbonate based electrolyte. However, it is incompatible with sulfur cathode. ${ }^{23}$ Thus, among the ether based electrolytes, the one containing $3 \mathrm{M}$ LiFSI and $1 \mathrm{M}$ LiTFSI in DME alone shows a much higher reversible capacity of $350 \mathrm{mAh} \mathrm{g}^{-1}$ over the commonly used DME/DOL based electrolyte which shows less than $300 \mathrm{mAh} \mathrm{g}^{-1}$, making it a more suitable choice for Li-S full cells. The compatibility and longevity of the MCMB-CP anode was further tested in this electrolyte. The DME electrolyte shows excellent reversibility over 100 cycles as evidenced in Figure 2c. Beyond the $1^{\text {st }}$ cycle, the Coulombic efficiency is about $99.8 \%$ establishing the stability of the SEI layer and that of the electrolyte with the anode. The specific capacity delivered by the cell drops to about $340 \mathrm{mAh} \mathrm{g}^{-1}$ in the second cycle, but stabilizes and still yields $327 \mathrm{mAh} \mathrm{g}^{-1}$ after 100 cycles. The voltage profile of the anode in Figure $2 d$ with the first discharge exhibits a short plateau at $0.8 \mathrm{~V}$ owing to the SEI layer formation. It also clearly shows the multi-step lithium intercalation into graphite. ${ }^{24}$ On lithium extraction, a reversible capacity of $350 \mathrm{mAh} \mathrm{g}^{-1}$ is obtained with a Coulombic efficiency of about $75 \%$ due to irreversible loss in the SEI formation. On further cycling, The Li insertion voltage settles to $0.09 \mathrm{~V}$ from cycle 10 to 100 . Li extraction occurs at $0.16 \mathrm{~V}$ at the $10^{\text {th }}$ cycle which increases to about $0.18 \mathrm{~V}$ by the $100^{\text {th }}$ cycle. Thus, the combination of $3 \mathrm{M} \mathrm{LiFSI}$ and $1 \mathrm{M}$ LiTFSI in DME yields a highly compatible electrolyte with the graphite anode. It has been shown that graphitic carbon paper can work as an effective anode in carbonate electrolyte. ${ }^{22,25}$ In the MCMB-CP anode, the capacity contribution from the carbon paper is about $2.18 \mathrm{mAh}$ and that of MCMB is 0.48 $\mathrm{mAh}$ thus providing a combined capacity of $2.66 \mathrm{mAh}$. Therefore, based on the sulfur in the cathode, there would be about $65 \%$ excess lithium on the anode providing sufficient capacity balance to operate a full cell while utilizing the full potential of sulfur.
Polysulfide cathode.-A novel approach to deposit solid lithium polysulfide in the cathode was developed in this work. It has been shown that polysulfide dissolved in the electrolyte works as a highly reversible sulfur source in a Li-S battery. ${ }^{26}$ It has also been shown that such a system is ideal for developing high sulfur loading cathodes. ${ }^{27}$ However, this approach limits the energy density as a large amount of electrolyte is required to deliver the sulfur. This can be circumvented by using the solvent evaporation technique utilized in this work. In this method, lithium polysulfide solution is prepared in a low boiling point media such as alcohol and deposited into a conductive substrate such as MWCNT paper and dried to remove the solvent leaving the polysulfide evenly distributed within the cathode. This process is outlined in the schematic in Figure $3 \mathrm{a}$ which shows the homogeneous $0.25 \mathrm{M}$ $\mathrm{Li}_{2} \mathrm{~S}_{6}$ solution in methanol and illustrates the cathode preparation technique. Such an approach also has the advantage of introducing extra lithium in the cathode thus minimizing the amount of lithium required in the anode. We utilized both $0.25 \mathrm{M}$ and $0.75 \mathrm{M}$ solutions of $\mathrm{Li}_{2} \mathrm{~S}_{6}$ to introduce polysulfide into the cell to demonstrate the viability of utilizing this technique to develop high polysulfide loading cathodes. The performance of the high loading cathode is shown in Figure 3 b. For the purpose of full cell study, the lower loading cathode was utilized due to the ease of fabrication of the anode with a lower loading of graphite at lab scale. However, this approach is scalable and can be utilized with higher loading of active materials on both the anode and cathode.

The absence of elemental sulfur or lithium sulfide in the cathode thus prepared was verified by XRD in Figure $4 \mathrm{a}$. The XRD of the
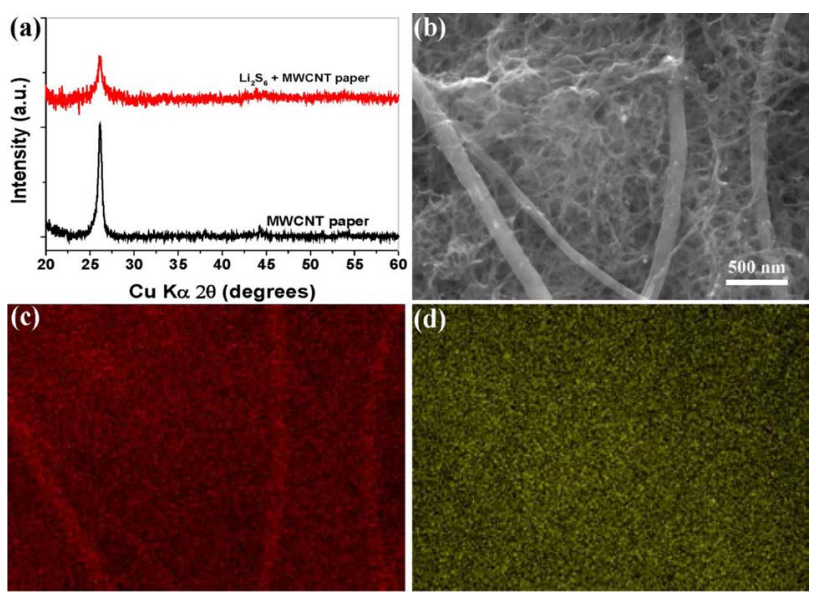

Figure 4. (a) XRD analysis of the polysulfide indicating amorphous deposits of polysulfide on the MWCNT paper, (b) SEM micrograph of the cathode along with EDX mapping of (c) carbon and (d) sulfur showing uniform coating of polysulfides throughout the cathode. 
(a)

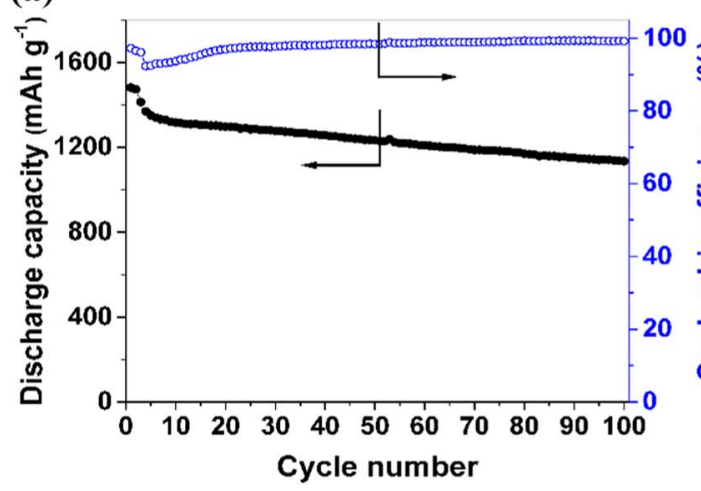

(b)

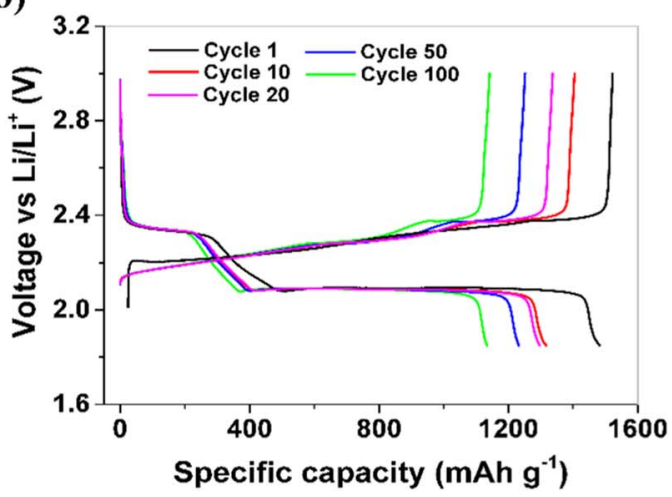

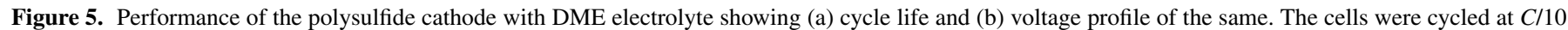
$\left(1 C=1,672 \mathrm{~mA} \mathrm{~g}^{-1}\right.$, based on the mass of sulfur in the polysulfide cathode).

cathode shows diminished peaks of the MWCNT paper thus proving that the cathode consists of an amorphous, conformal coating of lithium polysulfides. This was further confirmed through scanning electron microscopy (SEM). The SEM micrograph (Figure 4b) shows no distinct particles on the carbon network of the cathode. However, what appears to be a conformal coating of the lithium polysulfide species on the carbon is clearly observed. It is also possible that polysulfide species is absorbed by the pores of carbon nanotubes. The EDX mapping of carbon (Figure 4c) and sulfur (Figure 4d) confirm the uniform coating of polysulfide species in the cathode.

Half-cells were made with this cathode to examine its performance with the DME based electrolyte. The cycle life of the cell when cycled at $C / 10$ is shown in Figure 5a. During the initial cycles, the capacity drops from $1,481 \mathrm{mAh} \mathrm{g}^{-1}$ in the first cycle to $1,370 \mathrm{mAh} \mathrm{g}^{-1}$ in the $4^{\text {th }}$ cycle. The Coulombic efficiency drops to $93 \%$ in the same interval. This loss is probably due to minor polysulfide shuttle before the cell stabilizes. Beyond 20 cycles, electrochemical stability improves with the Coulombic efficiency reaching over $98 \%$. After 100 cycles, the cell manages to deliver over $1,100 \mathrm{mAh} \mathrm{g}^{-1}$ showing remarkable stability despite the absence of additives such as $\mathrm{LiNO}_{3}$. This demonstrates that the cathode prepared through this technique is robust in retaining most of the polysulfide generated during cell cycling within the pores of the cathode thus offering stable performance. The voltage profile of the cathode during first discharge shows the conversion of the high-order polysulfides $\left(\mathrm{Li}_{2} \mathrm{~S}_{\mathrm{x}}, 6 \leq \mathrm{x} \leq 8\right)$ to $\mathrm{Li}_{2} \mathrm{~S}_{4}$ above $2.1 \mathrm{~V}$ and its conversion to intermediate polysulfides $\left(\mathrm{Li}_{2} \mathrm{~S}_{\mathrm{x}}, 2 \leq \mathrm{x} \leq 4\right)$ at $2.1 \mathrm{~V}$ and lithium sulfide $\left(\mathrm{Li}_{2} \mathrm{~S}\right)$ at the end of discharge (Figure $\left.5 \mathrm{~b}\right)$. The following recharge converts the $\mathrm{Li}_{2} \mathrm{~S}$ to high-order polysulfides/elemental sulfur through the reversal of the discharge process. The charge process is able to reach completion beyond $2.4 \mathrm{~V}$ without leading to severe polysulfide shuttle. This highly reversible cycling is also captured by the cyclic voltammogram in Figure 6a. The continuous overlap of the peaks confirms the stability of the lithium polysulfide cathode with the new electrolyte. The high salt concentration of this electrolyte leads to higher viscosity thus inhibiting polysulfide dissolution and migration to the anode side. ${ }^{17,21}$ This capability of the electrolyte is demonstrated by its inability to form soluble polysulfide species on stirring of stoichiometric quantities of $\mathrm{Li}_{2} \mathrm{~S}$ and elemental $\mathrm{S}$ in a vial containing the electrolyte (Figure 6b). In comparison, as in Figure 6b, the common DME/DOL electrolyte is able to easily form polysulfide solution.

Full cell performance.-The excellent cycling stability offered by the cathode affords its use in the full cell setup. When coupled with the lithiated MCMB-CP anode and cycled at $C / 10$, the cycling performance in Figure $7 \mathrm{a}$ is observed. The initial formation cycles results in capacity decay from over $1,500 \mathrm{mAh} \mathrm{g}^{-1}$ to about $1,150 \mathrm{mAh} \mathrm{g}^{-1}$ as the anode and cathode performance stabilizes. Post stabilization, the full cell exhibits stable performance with Coulombic efficiency over $97 \%$. At the end of 100 cycles, about $700 \mathrm{mAh} \mathrm{g}^{-1}$ of the capacity is retained. In the first cycle (Figure 7b), as the cell discharges, 65\% excess lithium is present in the anode and thus operates at a lower potential ensuring high cell operating voltage. The high Li extraction

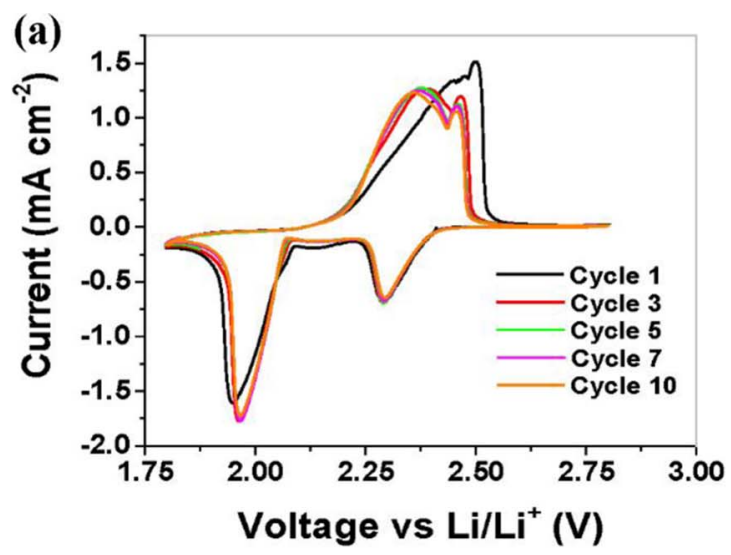

(b)

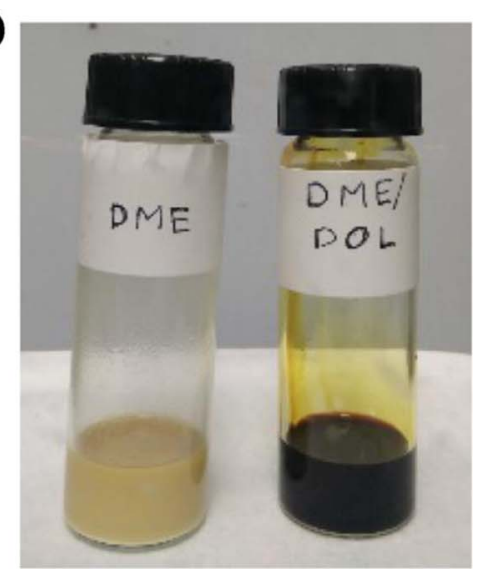

Figure 6. (a) Cyclic voltammogram of the polysulfide cathode showing its high reversibility, the scan rate is $0.1 \mathrm{mV} \mathrm{s}^{-1}$, (b) optical image demonstrating the relative insolubility of $\mathrm{Li}_{2} \mathrm{~S}_{6}$ in the DME electrolyte used in this work (left) and the ease of $\mathrm{Li}_{2} \mathrm{~S}_{6}$ formation in the conventional DME/DOL electrolyte. To prepare this, stoichiometric amounts of $\mathrm{Li}_{2} \mathrm{~S}$ and elemental sulfur was stirred in the electrolytes for $24 \mathrm{~h}$. 
(a)
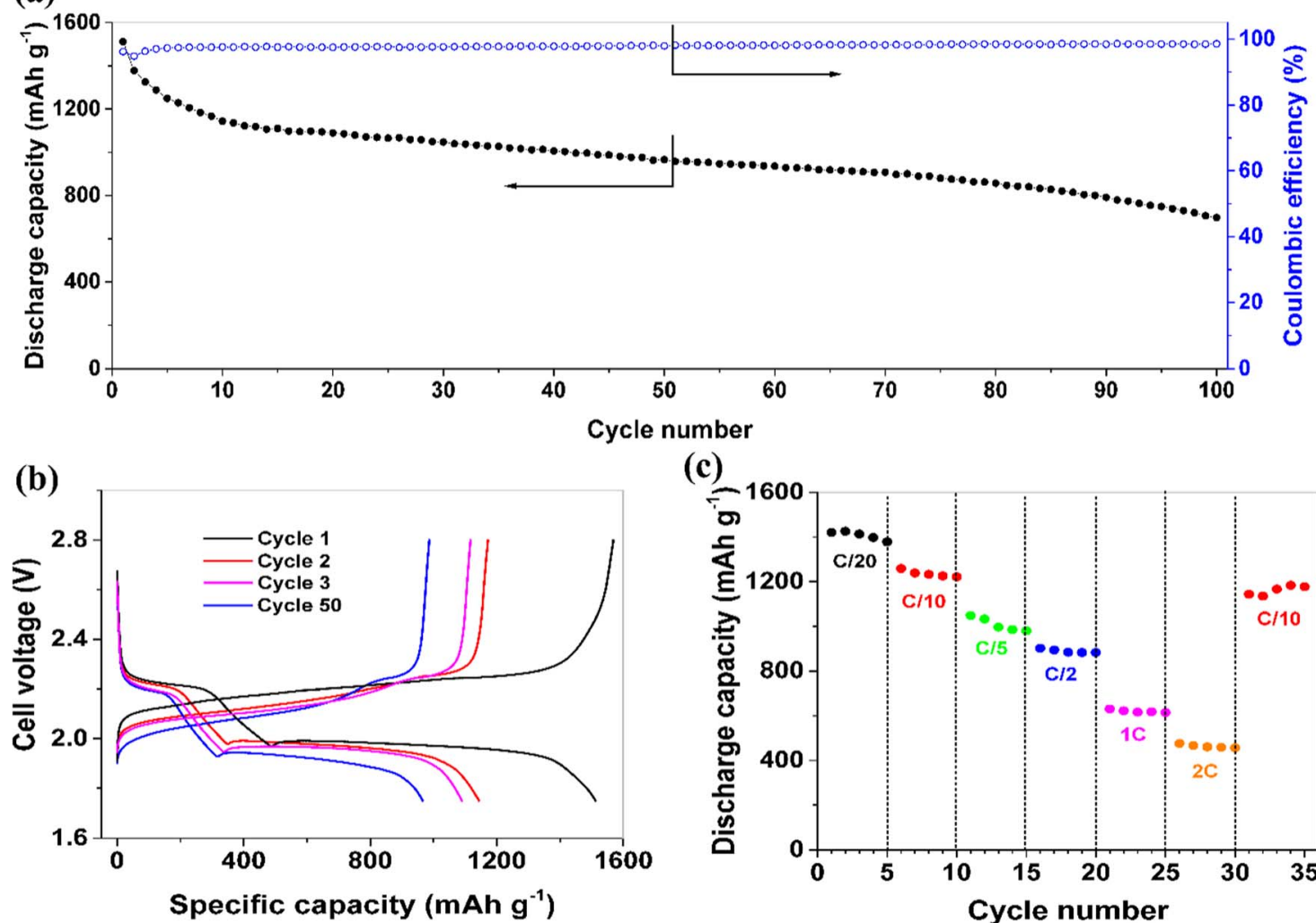

(c)

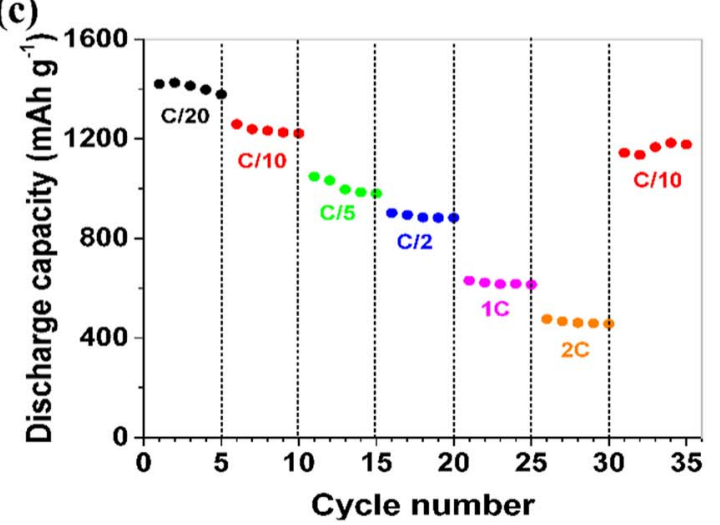

Figure 7. (a) Cycling performance of the full cell at $C / 10$ with its corresponding (b) voltage profile and (c) rate performance of the cathode. The cells were cycled at $C / 10\left(1 C=1,672 \mathrm{~mA} \mathrm{~g}^{-1}\right.$, based on the mass of sulfur in the cathode).

efficiency at the anode results in a high first discharge capacity of over $1,500 \mathrm{mAh} \mathrm{g}^{-1}$. The first cycle Coulombic efficiency is over $96 \%$. As the cycling progresses, both the discharge potential and the charge potential gradually lower, reducing the cell operating voltages. This is due to the consumption of lithium as the cycling progresses. This will be further discussed in the Post-mortem analysis section.

The full cell was tested using rates varying from $C / 20$ to $2 C$ to determine the reversible sulfur utilization at different rates with $1 C$ corresponding to a current of $1,672 \mathrm{~mA} \mathrm{~g}^{-1}$ sulfur (Figure 7c). At $C / 20$, a high reversible capacity of about $1,400 \mathrm{mAh} \mathrm{g}^{-1}$ is delivered by the cell. While cycling at $C / 10$, the cell delivers over $1,200 \mathrm{mAh}$ $\mathrm{g}^{-1}$. At higher rates of $C / 5, C / 2,1 C$, and $2 C, 1000 \mathrm{mAh} \mathrm{g}^{-1}, 880 \mathrm{mAh}$ $\mathrm{g}^{-1}, 610 \mathrm{mAh} \mathrm{g}^{-1}$, and $460 \mathrm{mAh} \mathrm{g}^{-1}$, respectively, can be extracted from the cell. On returning to $C / 10$, the cell is able to recover to the initial capacity level of $1,180 \mathrm{mAh} \mathrm{g}^{-1}$. This demonstrates excellent electrochemical reversibility of the cathode, enhanced Li diffusion through the anode and fast ion transport through the electrolyte. Thus, this full cell is able to deliver high capacities even under high rates.

Post-mortem analysis. - The substrate used in the cathode to deposit the polysulfide was commercial MWCNT paper that was used without any treatment. The primary contributor to the longevity of the cell is the ability of the MWCNT paper to contain the polysulfides. The cathode recovered from the cell after 100 cycles in the charged state (Figures $8 \mathrm{a}$ and $8 \mathrm{~b}$ ) is compared with that of the pristine cathode (Figures 8c and 8d). It is evident that, after 100 cycles, the MWCNT structure of the cathode still exhibits mechanical robustness and is able to accommodate the volume change occurring in the cathode. It also shows that the sulfur species is uniformly deposited and retained by the carbon network.

Although the electrolyte diminishes polysulfide solubility, some amount of it still diffuses over extended time which can be evidenced from the cycled anode. Comparison is made between an anode ex- tracted from a half cell after first cycle (Figures 9a, 9b, and 9c) and the anode extracted from a full cell after 100 cycles (Figures 9d, 9e, and 9f). The EDX map of both oxygen species (Figures $9 \mathrm{~b}$ and 9e) and sulfur species (Figures 9c and 9f) shed light on the two important reactions occurring at the anode, namely, SEI layer formation involving oxygen species arising from DME and the salts and sulfur migration due to shuttle effect. After first cycle, the anode shows
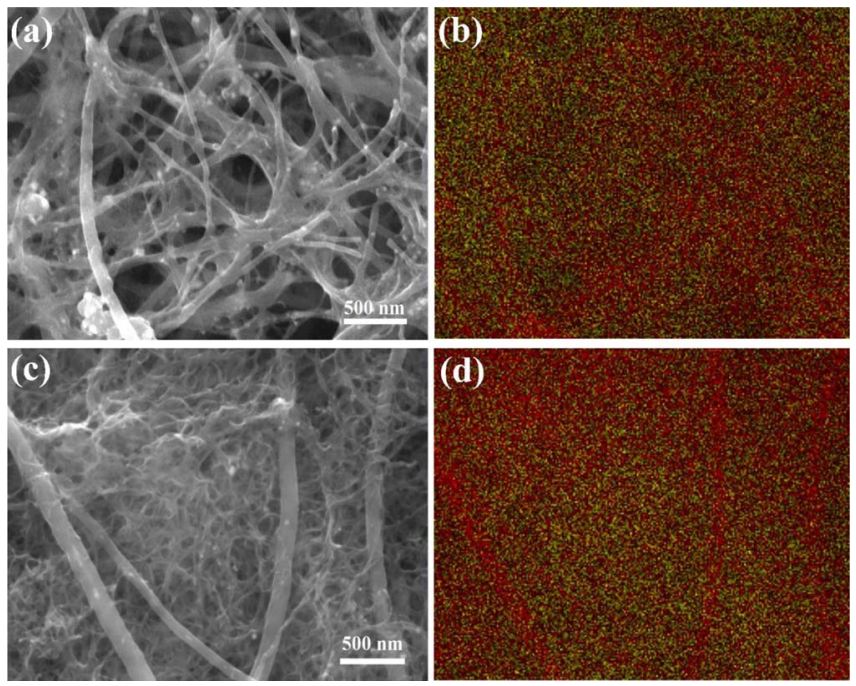

Figure 8. SEM image of the cathode (a) extracted after 100 cycles from a full cell accompanying its EDX mapping in (b) along with (c) as prepared cathode with its EDX mapping in (d). Red represents carbon and yellow represents sulfur. 

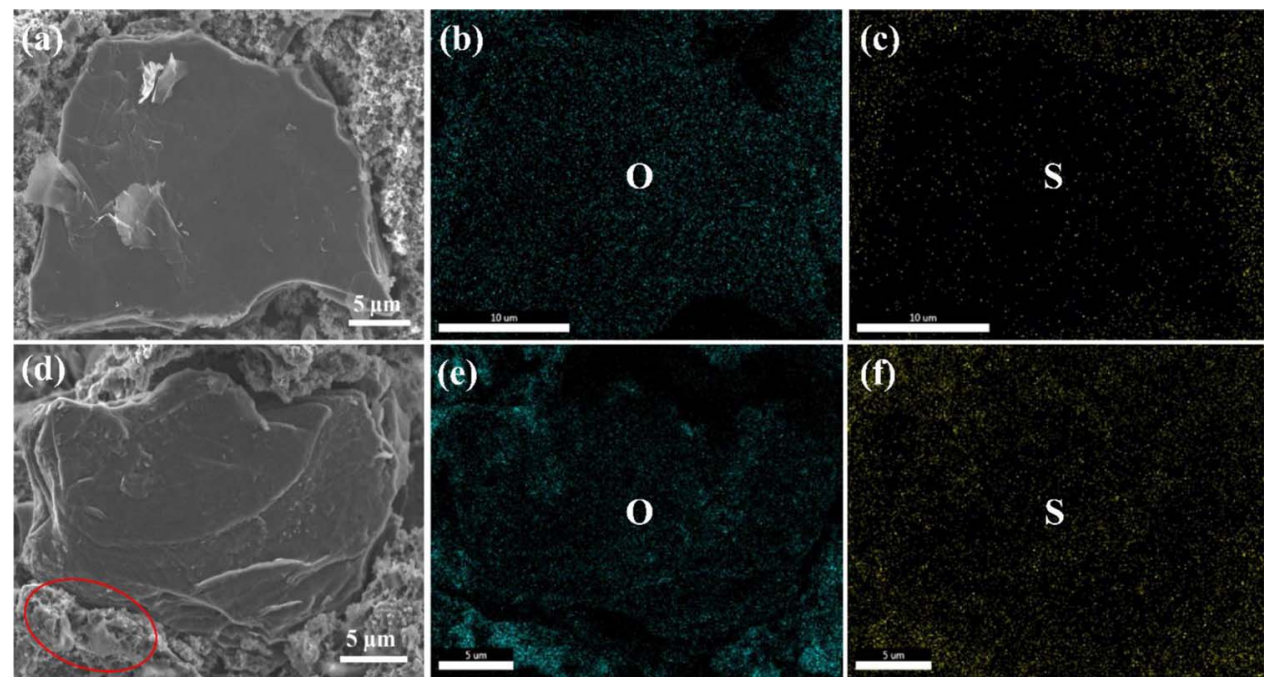

Figure 9. Post-mortem analysis of a MCMB-CP anode through (a) SEM micrograph along with EDX map of oxygen (b) and sulfur species (c) extracted from a half-cell compared with those of an anode from a cycled full cell (d), (e), and (f). Anodes were washed with DME solvent prior to analysis. The highlighted portion indicates insoluble sulfur species deposited on the anode.

a uniform, invisible formation of SEI on the surface of the MCMB particle as evidenced from the uniform distribution of oxygen species on graphite surface. There is almost no sulfur observed barring a small amount on the super C65 particles arising out of the decomposition of salts. This shows the formation of a uniform, robust SEI on the anode. In the cycled anode appears a distinct layer of deposits on both the MCMB and super C65 surface. The elemental mapping provides evidence on the nature of the deposit. It is most likely composed of sulfur species which on migration from the cathode, were reduced on the anode surface and deposited there. This process corrodes the SEI layer leading to its reformation through electrolyte decomposition. The high sulfur concentration in the deposit highlighted in Figure 9d supports this hypothesis.

Therefore, the polysulfide shuttle, although not severe, leads to the loss of capacity over cycles. As there still exists a minor shuttle effect, the migrating polysulfides slowly consume lithium at the anode and form deposits of $\mathrm{Li}_{2} \mathrm{~S}_{2} / \mathrm{Li}_{2} \mathrm{~S}$. As ten or more moles of $\mathrm{Li}$ is required per mole of $\mathrm{Li}_{2} \mathrm{~S}_{6}$ (or higher order) that migrate to the anode side, the excess lithium present in the anode depletes fast leading to the operation of the anode at higher potential. This reduces the cell voltage as observed in Figure 7b. The performance of such a system could be enhanced by modification of the polysulfide host such as functionalization of $\mathrm{CNT},{ }^{28}$ modification of current collectors, ${ }^{29}$ use of other host materials such as graphene, ${ }^{30}$ ordered carbon, ${ }^{31}$ and other non-carbonaceous hosts to contain lithium polysulfides in the cathode. $^{32}$

\section{Conclusions}

A major factor slowing the progress of Li-S batteries toward commercialization is the lack of a viable alternative to the lithium metal anode. The development of electrolytes that can provide stable, long term cycling with non-lithium metal anodes while maintaining compatibility with sulfur cathode is an alternative direction toward the progress of Li-S batteries. In this work, as a model study, we have utilized a graphitic anode consisting of MCMB embedded in carbon paper that demonstrates stable lithium intercalation and de-intercalation through the formation of a robust SEI while using $3 \mathrm{M} \mathrm{LiFSI}$ and $1 \mathrm{M}$ LiTFSI in DME as the electrolyte. This unique electrolyte combination enhances the compatibility with sulfur cathode. We also report a novel technique for solid lithium polysulfide introduction into a carbon current collector. On coupling this cathode with the graphitic anode in the new electrolyte system, a stable, high performance full cell is obtained. This work demonstrates the viability of utilizing this approach of cathode and anode design to increase active material loading and cycle life through material optimization which can work in tandem with the new electrolyte to make a long life Li-S full cell.

\section{Acknowledgments}

This work was supported by the startup grant from Purdue School of Engineering and Technology and Department of Mechanical Engineering at Indiana University-Purdue University Indianapolis. We acknowledge the Integrated Nanosystems Development Institute (INDI) for use of their Bruker D8 Discover XRD Instrument, which was awarded through the NSF grant MRI-1429241 and for use of their JEOL7800F Field Emission SEM, which was awarded through NSF grant MRI-1229514.

\section{References}

1. J. Lim, J. Pyun, and K. Char, Angew. Chem. Int. Ed., 54, 3249 (2015).

2. A. Manthiram, Y. Fu, S.-H. Chung, C. Zu, and Y.-S. Su, Chem. Rev., 114, 11751 (2014).

3. A. Manthiram, Y. Fu, and Y.-S. Su, Acc. Chem. Res., 46, 1125 (2012).

4. S. S. Zhang, J. Power Sources, 231, 153 (2013).

5. H. Kim, G. Jeong, Y.-U. Kim, J.-H. Kim, C.-M. Park, and H.-J. Sohn, Chem. Soc. Rev., 42, 9011 (2013).

6. D. Lu, Y. Shao, T. Lozano, W. D. Bennett, G. L. Graff, B. Polzin, J. Zhang, M. H. Engelhard, N. T. Saenz, W. A. Henderson, P. Bhattacharya, J. Liu, and J. Xiao, Adv. Energy Mater., 5, 1400993 (2015).

7. D. Aurbach, E. Pollak, R. Elazari, G. Salitra, C. S. Kelley, and J. Affinito, J. Electrochem. Soc., 156, A694 (2009).

8. R. Cao, W. Xu, D. Lv, J. Xiao, and J.-G. Zhang, Adv. Energy Mater, 5, 1402273 (2015).

9. M. Agostini, B. Scrosati, and J. Hassoun, Adv. Energy Mater, 5, 1500481 (2015).

10. S. Zheng, Y. Chen, Y. Xu, F. Yi, Y. Zhu, Y. Liu, J. Yang, and C. Wang, ACS Nano, 7, 10995 (2013).

11. D. Lv, P. Yan, Y. Shao, Q. Li, S. Ferrara, H. Pan, G. L. Graff, B. Polzin, C. Wang, and J.-G. Zhang, Chem. Commun., 51, 13454 (2015).

12. J. Brückner, S. Thieme, F. Böttger-Hiller, I. Bauer, H. T. Grossmann, P. Strubel, H. Althues, S. Spange, and S. Kaskel, Adv. Funct. Mater, 24, 1284 (2014).

13. M. Hagen, E. Quiroga-González, S. Dörfler, G. Fahrer, J. Tübke, M. J. Hoffmann, H. Althues, R. Speck, M. Krampfert, S. Kaskel, and H. Föll, J. Power Sources, 248, 1058 (2014).

14. S.-K. Lee, S.-M. Oh, E. Park, B. Scrosati, J. Hassoun, M.-S. Park, Y.-J. Kim, H. Kim, I. Belharouak, and Y.-K. Sun, Nano Lett., 15, 2863 (2015).

15. N. Moreno, M. Agostini, A. Caballero, J. Morales, and J. Hassoun, Chem. Commun., 51, 14540 (2015).

16. Y. Yamada and A. Yamada, J. Electrochem. Soc., 162, A2406 (2015).

17. L. Suo, Y.-S. Hu, H. Li, M. Armand, and L. Chen, Nat. Commun., 4, 1481 (2013).

18. E. S. Shin, K. Kim, S. H. Oh, and W. I. Cho, Chem. Commun., 49, 2004 (2013).

19. R. Miao, J. Yang, X. Feng, H. Jia, J. Wang, and Y. Nuli, J. Power Sources, 271, 291 (2014). 
20. J. Hu, G. Long, S. Liu, G. Li, and X. Gao, Chem. Commun., 50, 14647 (2014).

21. H. Kim, F. Wu, J. T. Lee, N. Nitta, H. T. Lin, M. Oschatz, W. I. Cho, S. Kaskel, O. Borodin, and G. Yushin, Adv. Energy Mater, 5, 1401792 (2015).

22. Y. Fu and A. Manthiram, Nano Energy, 2, 1107 (2013).

23. J. Gao, M. A. Lowe, Y. Kiya, and H. D. Abruña, J. Phys. Chem. C, 115, 25132 (2011)

24. Z. Jiang, M. Alamgir, and K. M. Abraham, J. Electrochem. Soc., 142, 333 (1995).

25. A. Bhargav and Y. Fu, J. Electrochem. Soc., 162, A1327 (2015).

26. Y. Fu, Y.-S. Su, and A. Manthiram, Angew. Chem. Int. Ed., 52, 6930 (2013).
27. L. Qie, C. Zu, and A. Manthiram, Adv. Energy Mater, 1502459 (2016).

28. J. Yang, J. Xie, X. Zhou, Y. Zou, J. Tang, S. Wang, F. Chen, and L. Wang, J. Phys Chem. C, 118, 1800 (2014)

29. Y. Cui and Y. Fu, ACS Appl. Mater. Interfaces, 7, 20369 (2015).

30. H. Wang, Y. Yang, Y. Liang, J. T. Robinson, Y. Li, A. Jackson, Y. Cui, and H. Dai, Nano Lett., 11, 2644 (2011).

31. X. Ji, K. T. Lee, and L. F. Nazar, Nat. Mater, 8, 500 (2009).

32. Q. Pang, D. Kundu, and L. F. Nazar, Mater. Horiz, 3, 130 (2016). 